\title{
Barriers and Facilitators to Implementing Evidence Based Bleeding Management in Australian Cardiac Surgery Units: A Qualitative Interview study analysed with the Theoretical Domains Framework and COM-B model
}

Bronwyn Louise Pearse ( $\square$ bronwyn.pearse@health.qld.gov.au )

Prince Charles Hospital https://orcid.org/0000-0003-0048-620X

\section{Samantha Keogh}

Queensland University of Technology

\section{Claire M Rickard}

Griffith University Menzies Health Institute Queensland

\section{Yoke Lin Fung}

University of the Sunshine Coast

Research article

Keywords: Cardiac surgery, Australia, Bleeding, Barriers, Facilitators, Theoretical Domains Framework, COM-B Model

Posted Date: November 10th, 2020

DOI: https://doi.org/10.21203/rs.3.rs-27829/v2

License: (1) This work is licensed under a Creative Commons Attribution 4.0 International License.

Read Full License

Version of Record: A version of this preprint was published at BMC Health Services Research on June 5th, 2021. See the published version at https://doi.org/10.1186/s12913-021-06269-8. 


\section{Abstract}

\section{Background}

Bleeding during cardiac surgery is a common complication that often requires the transfusion of blood products. The combination of bleeding and blood product transfusion incrementally increases adverse outcomes including infection and mortality. Following bleeding management guideline recommendations could assist with minimising risk but adherence is not high, and the cause for lack of adherence is not well understood. This study aimed to identify barriers and facilitators to practicing and implementing evidenced-based intra-operative, bleeding management in Australian cardiac surgery units.

Methods

We used a qualitative descriptive design to conduct semi-structured interviews with Australian cardiac surgeons, anaesthetists and perfusionists. The Theoretical Domains Framework (TDF) was utilised to guide interviews and thematically analyse the data. Categorised data were then linked with the three key domains of the COM-B model (capability, opportunity, and motivation) to explore and understand behaviour.

Results

Seventeen interviews were completed. Nine of the 14 TDF domains emerged as significant. Analysis revealed key themes to improving capability included, standardisation, monitoring, auditing, and feedback of data and cross discipline training. Opportunity for change was improved with interpersonal and interdepartmental collaboration through shared goals, and more efficient and supportive processes allowing clinicians to navigate unfamiliar business and financial models of health care. Results suggest as individuals, clinicians had the motivation to make change and healthcare organisations have an obligation and a responsibility to partner with clinicians to support change and improve goal directed best practice.

Conclusion

Using a theory-based approach it was possible to identify factors which may be positively or negatively influencing clinicians ability to implement best practice bleeding management in Australian cardiac surgical units.

\section{Background}

Excessive bleeding occurs during and after cardiac surgery in up to $10 \%$ of patients; and the most common treatment is the transfusion of blood products $(1,2)$. However, blood transfusion is a form of tissue allotransplantation associated with circulatory overload, acute lung injury, febrile and allergic and haemolytic reactions, alloimmunisation, immunomodulatory effects, and bacterial and infectious disease transmission (3-6). Furthermore, the combination of excessive bleeding, and management with blood 
transfusion and re-exploration surgery incrementally increases adverse outcomes including infection and mortality (6-13).

Many evidence-based, guideline-supported strategies exist to manage bleeding, including the collaborative contribution of the surgical team (14-18). In 2010, the implementation of multidisciplinary and multimodal concepts to manage bleeding and blood loss were endorsed by the World Health Organisation (WHO) as part of the Patient Blood Management paradigm (PBM)(19). However, the complex and dynamic context of cardiac surgery makes the implementation of bleeding management a genuine challenge for the surgical team. This is well documented in the literature noting considerable variation in bleeding management practice and outcomes $(1,20-23)$.

Although health care professionals are expected to implement strategies that provide best outcomes, the required knowledge and skills to change practice are often not a core component of health care professional training (24). Difficulties with implementation may be compounded by factors relating to the environment, context, resources, and social influences (25-27). As a result, clinicians may not always provide evidence-based bleeding management, even when they perceive patients would benefit from such interventions.

It is known from behavioural theories that clinicians must have the capability to improve practice or make change, as well as the opportunity and motivation to do so (28). In the cardiac surgical setting, the barriers and facilitators to the implementation and provision of evidence-based bleeding management are unclear. Previous research has focused on outcomes of bleeding including the transfusion of blood products, rather than focussing upstream, investigating management of the bleeding episode. Consequently, there are important gaps in knowledge regarding clinicians' ability to implement and provide best practice bleeding management. It is therefore necessary to investigate and understand more about this area of practice and the implications on health care outcomes. The aim of this qualitative study was to identify barriers and facilitators that surgeons, anaesthetists and perfusionists face when practicing and implementing evidenced-based, intra-operative bleeding management in Australian cardiac surgery units.

\section{Methods}

\section{Design}

This study employed a qualitative design to investigate the perceptions of barriers and facilitators to practicing and implementing bleeding management in Australian cardiac surgery units. Semi-structured interviews with cardiac surgeons, cardiac anaesthetists and perfusionists were undertaken in November and December 2018.

Theoretical Framework 
We employed two theoretical models of behaviour change to provide both theoretical and pragmatic guidance for this study: 1. the Theoretical Domains Framework (TDF), and 2. the Capability, Opportunity and Motivation Behaviour Model (COM-B)(29). The TDF is a framework that can be used in a theory based evaluation to identify factors (i.e. barriers and facilitators) that may influence behaviour. The TDF is a 'first step' to identify and categorise a given 'barrier and/or facilitator' to behaviour rather than an explanation of how change takes place. The 14 validated TDF domains are: 1. knowledge, 2. skills, 3. social/professional role and identity, 4. beliefs about capabilities, 5 . optimism, 6 . beliefs about consequences, 7. reinforcement, 8. intentions, 9. goals, 10. memory, attention, and decision processes, 11. environmental context and resources, 12. social influences, 13.emotion, and 14. behavioural regulation. The interview topic guide was based on the 14 domains to view and categorise cognitive, affective, and social and environmental influences on behaviour. The TDF-based interview topic guide was developed and refined through discussion with the study team and pilot testing with a surgeon, an anaesthesiologist and perfusionist. Open-ended questions were used, and the number of questions ranged from one to three for each TDF domain. Follow-up prompts were included when necessary to address specific constructs within the domains. Data categorised with the TDF framework were distilled and linked with the three key domains of the COM-B model, then used to analyse and understand behaviour. The COM-B model of behaviour can be used to identify what needs to shift or be modified, so that behaviour change interventions can be effective. The model identifies three components that need to be present for behaviour to occur: capability, opportunity, and motivation. Capability covers a person's psychological and physical capacity to adopt a particular behaviour. Opportunity relates to all the external factors that make a behaviour possible. Motivation covers the thought processes that direct behaviour. Both capability and opportunity can influence motivation. Therefore, while motivation addresses whether clinicians will or won't adopt a particular behaviour; capability, and opportunity address whether a clinician 'can or can't adopt it. These components are dynamic and interact over time where behaviour can be seen as part of a system with positive and negative feedback loops(30-32).

\section{Participants}

Participants who took part in the study were those clinicians involved in the direct management of intraoperative bleeding and included cardiac surgeons, cardiac anaesthesiologists and clinical perfusionists. A combination of convenience and snowball sampling techniques were used to recruit 17 clinicians from a previous national cross-sectional survey on bleeding management practice by the same authors(33). Participants from that survey were asked to email their interest in participating in a future qualitative interview study and participants emerged through a process of reference. Based on the threshold for data saturation in previous studies with health care clinicians and using the TDF, we projected that 12 to 18 interviews would be required (34). Final sample size was again determined through data saturation, which was considered to have occurred when no new data were identified in three successive interviews. Invitation letters, study information sheets and a consent form were sent via email inviting 23 clinicians to participate in interviews. If no response was received after a week, a second email was sent. The primary reason for non-participation was lack of time or scheduling issues. 


\section{Data collection}

Brief introductions took place before the consent that was emailed to participants at recruitment was reviewed and signed prior to the interview. In depth interviews were conducted by the lead author and were audio recorded. Open-ended questions were used to encourage participants to explore their experiences and specific instances with managing or implementing bleeding management including barriers and facilitators (35).

\section{Data Analysis}

For the purpose of consistent coding, a coding guide was developed based on the published definitions and concepts of the TDF domains (Appendix 1). The 17 audio-recorded interviews were transcribed verbatim and imported into the qualitative data analysis software package NVivo 12 (QSR International). Excerpts were coded into the main domains of the TDF using theory-based content analysis by the first author (BP). In the next stage, specific beliefs, including both barriers and facilitators were collated, defined, and grouped into sub-themes under each domain. The NVivo software organises data into number of references or occurrences, attributable to participants. Further analyses were also performed to compare interviewees' statements by hospital type (public/private, metropolitan/regional). A second and third member (YLF and SK) independently analysed the interviews (approximately $65 \%$ and 35\% respectively), to ensure reliability of the coding guide. All coding was discussed and agreed upon by the study team. The research team met several times to refine categories and clarify any issues. Memos were used to record relevant discussions and coding notes.

Criteria were developed to determine which domains of the TDF were 'relevant': 1 . qualitatively; where specific beliefs were coded frequently within a domain and 2. quantitatively; where domains contained strong beliefs regarding barriers/facilitators to practicing and implementing provide evidence-based bleeding management. Relevance was considered to be achieved when domains met both criteria.

\section{Member Checking}

Following the data analyses, all participants were given the opportunity to review the synthesised member check document. Member checking provided participants with the opportunity to add clarification or new information and prioritise the inferences, emerging concepts, and initial themes.

\section{Results}

Participants (cardiac surgeons, $n=5$, cardiac anaesthesiologists, $n=7$, clinical perfusionists, $n=5$ ) represented key stakeholders involved in practicing and implementing bleeding management in cardiac surgery in Australia. Participants reported practicing from 9 to 29 years, and practiced across different states and settings including public (6), private (1) and both (10). Length of interviews ranged from 27 
minutes to 53 minutes. Specific participant demographics or any identifying data within the quotes were not included to protect anonymity.

Of the 14 TDF domains, nine emerged as significant to categorise clinicians' beliefs about the barriers and facilitators to managing bleeding and implementing change to improve practice. Four TDF domains were present but rare. (Table 1)

Table 1 TDF Domains reported by occurrence and participants

\begin{tabular}{|c|c|c|c|c|}
\hline COM-B & TDF & $\begin{array}{l}\text { No. of } \\
\text { Occurrences }\end{array}$ & $\begin{array}{l}\text { No. of } \\
\text { Participants }\end{array}$ & $\begin{array}{l}\% \\
\text { Participants }\end{array}$ \\
\hline & $\underline{\text { Relevant Domains }}$ & & & \\
\hline \multirow[t]{3}{*}{ Capability } & Behavioural Regulation & 54 & 14 & $82 \%$ \\
\hline & Knowledge & 77 & 13 & $76 \%$ \\
\hline & Skills & 23 & 12 & $71 \%$ \\
\hline \multirow[t]{2}{*}{ Opportunity } & $\begin{array}{l}\text { Environmental Context and } \\
\text { Resources }\end{array}$ & 81 & 17 & $100 \%$ \\
\hline & Social Influences & 109 & 16 & $94 \%$ \\
\hline \multirow[t]{5}{*}{ Motivation } & Belief about Capabilities & 34 & 14 & $82 \%$ \\
\hline & $\begin{array}{l}\text { Social Professional Roles \& } \\
\text { Responsibility }\end{array}$ & 47 & 14 & $82 \%$ \\
\hline & Belief about Consequences & 25 & 12 & $71 \%$ \\
\hline & Emotion & 17 & 10 & $59 \%$ \\
\hline & Rarely Reported Domains & & & \\
\hline Capability & $\begin{array}{l}\text { Memory, Attention, and } \\
\text { Decision Making }\end{array}$ & 4 & 4 & $23 \%$ \\
\hline \multirow[t]{3}{*}{ Motivation } & Intentions & 4 & 4 & $23 \%$ \\
\hline & Goals & 5 & 3 & $18 \%$ \\
\hline & Optimism & 7 & 3 & $18 \%$ \\
\hline
\end{tabular}

A number of explanatory themes (barriers and facilitators) were connected within and across domains, while remaining a specific theme (illustrated by connecting arrows in Figure 1.

\section{Capability: Psychological Capability, Physical Capability}

'Capability' (COM-B) can be explained as the clinician's capacity to engage in the management of actual bleeding episodes or implement change to improve practice. Six barriers and three facilitators emerged in relation to this construct. 


\section{Behavioural Regulation (psychological capability)}

These included three related to "Behavioural Regulation" (TDF) whereby clinicians described standardisation with protocols and decision support tools as useful to guide and reduce variation in practice. All quotations are followed by recognition of profession (S) for surgeon, $(A)$ anaesthetist, $(P)$ perfusionist.

"I think that protocol-based practice has a huge amount going for it - reproducibility of what you do, patient safety, everyone being able to be on the same page every time. I think that the answer to a lot of these problems, is having protocols to drive your practice" $(A)$

"So, to follow a protocol and get the majority of problems (bleeding) sorted - 95-99\% of the time, is really easy" (A)

"If it's done every time, it's part of the vernacular... it becomes part of the language. It has to become part of the standard practice, so it's more habit actually, that changes" (A)

"An algorithmic guideline helps you remember things that you may have otherwise forgotten" (A)

However, there was also the belief that flexibility in decision making using experience were equally important because of locally contextual clinical and environmental issues.

"It's a combination of evidence based and personal preference and personal experience" (S)

"It's never going to be a rigid application of guideline for every single patient and there has to be that of course" (A)

"It's like a mental check list but it's dependent on the patient, the procedure, where I'm operating and who I'm working with" (S)

"One of the problems with clinical medicine is that people do tend to think dichotomously rather than continuously and that's not helpful" (A)

Additionally, participations considered behaviours and the ability to implement improvement could be influenced by audit and feedback This was considered particularly relevant as surgeons and anaesthetists were highly driven, often competitive, and capitalising on these traits was considered an enabler for practice improvement.

"It's about incentive, we have brought it in for them....... the de-identified data, where they all want to be like everybody else. They don't want to be an outlier, so when you can present them with a graph with their de-identified, they take note" (A)

"It's important to have feedback loops because you become accustomed to what you are doing whether its adequate or inadequate" $(P)$ 
"I think training, education and quality assurance provided by having dedicated CNCs' support for blood management is essential and this feeds our knowledge and that feeds into audit data and that in turn feeds back into proving efficiencies and effectiveness" (A)

"There is a big difference between what people know and what they practice and without the collection of data your opinion can be enormously skewed to fit your own belief system" (A)

\section{Knowledge (psychological capability) and Skills (physical capability)}

Four themes emerged related to the TDF domain 'knowledge', two related to 'skills'specifically and two themes bridging both domains. (Table 1) In the first bridged theme, participants supported the concept of joint educational opportunities with relevant colleges and societies to improve both 'knowledge' and 'skills' within the multidisciplinary framework. There was a belief that this type of joint training could address the lack of a common language.

"Collaborative teaching would be helpful. It has to be multi-disciplinary though, so you can't have the cardiac ANZSCTS (Australian \& New Zealand Society of Cardiothoracic Surgeons) doing one thing for the cardiac surgeons and ANZCA's (Australia and New Zealand College of Anaesthetists) cardiac special interest group doing something for the cardiac anaesthetists and they're different. You've got to be working from the same knowledge base" (A)

"An area where both groups can get together and learn and have a combined approach. I think team management is important so that when we say to the surgeon, $X$ and $Y$ are ok but maybe you should consider $Z$, but they say to us but $A, B$ and $C$. We're both talking the same language and making a collective decision based on that" $(A)$

In the second bridged theme participants overwhelmingly reported 'skills' and 'knowledge' were acquired informally by clinicians with a particular interest in bleeding management, then learnings passed in an ad hoc way or, on the job discussion.

"Peripherally, there are bits of knowledge you can gain and obtain, but I'm not aware of a specific course or a specific online teaching resource for bleeding in cardiac surgery" $(A)$

"Currently you have knowledge that is dispensed by individuals, it is uncoordinated, based on opinion, and I think that is part of the confusion with blood management" $(P)$

"I do think that the bleeding that you see in cardiac surgery is unique and the patterns of coagulopathy that you see associated with cardiac surgery are unique and that trying to lump those in with other major bleeding and other surgery or trauma is a mistake" $(A)$

The majority (but not all) participants believed that specialist training could be improved with the inclusion of more up to date learning for example, related to the cell-based model of coagulation, 
viscoelastic haemostatic assays and goal directed therapy.

"I never had any formal teaching in haemostasis except those diagrams in medical school that actually mean nothing the, INR and PT and APTT. I don't think any of those tests are useful in bleeding management" $(S)$

"I think it needs to get into the training programs. It needs to become second nature for people. We're sort of attacking it from the wrong end trying to grab people by the time they're out and invested in their current practice" $(A)$

"I think there is probably a very variable range of knowledge among cardiac anaesthetists about management of coagulation, to be honest with you" (A)

"In my training, I got taught very little about bleeding. Not at medical school and not at surgical school, so when we started doing blood management here, I knew a bit about bleeding and haemostasis management but I didn't know a lot and I didn't know anything about ROTEM or TEG or any of those technologies and so I had to learn" (S)

"Every cardiac surgeon during its training is well-experienced. I think in six years they know how to control bleeding and how to manage patients with bleeding" (S)

"Anaesthetists probably have the most education on managing major haemorrhage and bleeding, we have to do major haemorrhage and critical bleeding modules for CPD" (A)

"...they are still being taught these old pathways that exist in test tubes and not being taught practical stuff in terms of bleeding management" $(S)$

A barrier to 'knowledge' was widespread acknowledgement of non-compliance with current guidelines. Deviations from recommendations were considered an accepted part of practice. It was generally believed this was due to divergent recommendations, recommendations based on low levels of evidence, and a lack of local relevance.

"I am aware of multiple sets of guidelines through my own work and research. They don't all harmonise of course, and so that's problematic" (A)

"They (Australian \& New Zealand Society of Cardiothoracic Surgeons) would be an authority that I would turn to because they're local, but they don't have anything specific" (A)

"The challenge that we have for example, the European guidelines, there is a lot of factor concentrates and they have different systems, so they don't necessarily apply to here" (A)

"The problem being of course is that they (guidelines) are disparate in some respects. On occasions, the guidelines actually contradict each other, or else they have different weightings for the level of evidence that they present and that of course is a problem" (A) 
Participants felt that they often lacked the skills needed to negotiate and relate with finance/business departments when trying to introduce new equipment, testing, products, or additional clinical time to support practice improvement. It was felt that, not only the language used by clinicians, but the goals and outcomes of these different departments did not align and was a source of frustration.

"In the public hospital system, a huge barrier is that doctors and medical people are not trained in the same way in business and in budgeting and even how to interact with groups outside the medical profession" (A)

"The project management skills that you need to set up a program are quite significant, and I think many of us, not only don't have the time to do it, but don't have the skills unless you have them intrinsically, they are not there" (A)

"You've got to learn how to talk to all these other people, the funders and the bean counters, you've got to learn to talk to them in a way that they understand and sell yourself" (A)

Opportunity: Physical Opportunity, Social Opportunity

'Opportunity' (COM-B) can be explained as all the factors that lie beyond the individual, that make the management of bleeding or implementation of practice improvement, possible. These included factors in the environment that encourage or discourage these goals. These factors can be physical for example, time constraints, resources, cost, physical environmental barriers. Or they can be related to social context of practice including interpersonal, intradepartmental, or interdepartmental influences, group or individual attitudes, culture, or the expectations of others. Five barriers and one enabler emerged as influencing participants within this construct. These included three related to three related to 'Environmental context and resources' and two related to 'Social influences'.

\section{Environmental Context and Resources (physical opportunity)}

Participants believed that complicated administrative processes negatively influenced their ability to implement improvements in bleeding management. Compounding these barriers were a lack of organisational and/or managerial support and leadership for training/education, skill development, resources, and dedicated blood management clinicians. Participants also reported considerable time restraints due to their primary clinical responsibility with little or no time to dedicate to write business cases for funding, or develop processes, policies, tools, and educational packages required to change practice.

"We've got pain nurses and infection-control nurses, we've got joint-care nurses, we've got colostomy nurses, but we don't have a blood management nurse who is here every day like those others, who can do this stuff. I'll know that there is an issue but it's going to take me two weeks until I can actually sit down and make an attempt to address it. A person who had the time, dedicated to this sort of thing, would really make a big difference" (A) 
"Putting a business case together, no body teaches you how to do that and it takes a lot of time. We're clinicians looking after patients, who has the time?" (A)

"You need to have both support from the Chief Executive as well as the individual people in the room. I think all are very important" $(S)$

"Administrative bureaucracy that prevents improvements from happening. That's one thing you have to overcome. I think that's definitely a big barrier, it's frustrating" (A)

"The external factors, non-clinical entities, which affect us, I don't think that anybody would stand in the way of us delivering evidence-based care on a day to day basis but they will stand in the way of providing the resources that we need to do it over the short to medium long term" (A)

Participants agreed that differences in resourcing between private/public \& regional/ metropolitan hospitals influenced bleeding management and the ability to implement practice improve initiatives.

"It's difficult, I can't really say, 'let's not give anything' because we don't have any evidence without a ROTEM or access to platelet function, and I am not the one who has a bleeding patient under my hands. I think we have some power, to discuss and talk but no real time information to make treatment decisions and that limits our input" $(A)$

"I don't have a good answer for how it would work in the private sector, but the public sector you have a chance of getting a paid person, generally like a nurse educator type role, that works best I think in the public sector" $(A)$

"..... it's less easy, for instance, simple things like getting blood. You can wait hours for blood or platelets at some hospitals" (S)

"In private it makes it more difficult because you don't really know what the status of the patient is, because there is no ROTEM to go by" $(P)$

"Something like a research nurse that would enable the data collection and the day to day management and the education of people, I think, is probably the tool that is best suited to do it in the public sector" (A)

"Sometimes I can't get what I need because sometimes there are resource limitations. Sometimes specific hospitals don't have specific products, especially the private ones that are smaller" (A)

\section{Social Influences (social opportunity)}

A very specific barrier participants discussed was the conflicting processes, goals, and outcome criteria from the pathology and haematology departments that had a direct impact on clinician's ability to make change improvements. 
"Pathology and other departments who have various policies in place that make it difficult to implement change to begin with. It can be discouraging" (A)

"If I wanted to bring that in, I would have to sit with haematology, and they are concerned that I don't get a rebate, or they don't get paid enough to do platelet function tests. They are not interested in doing it because it will be cost for their department, without money for their department because the savings are in the blood bank which is a different department. That's an external factor. That is a problem" (A)

"There was a resistance for a long time from haematology to basing any decisions on viscoelastic testing" (A)

"A haematologist who hasn't quite got their head around that... we care about this and we don't want to use blood products inappropriately. We don't want to treat with a therapy that just isn't needed, not necessary. So that's our brick wall, that's our problem here, we can't get around that" $(A)$

"Private pathology is not supporting that sort of stuff, because there is no reason really for them to do it" (A)

Participants overwhelming recognised that effective teamwork, collaboration, and communication between disciplines was critical for successful implementation and management of bleeding.

"I think the things most contingent on managing bleeding, are the roles and relationships between the clinicians" $(P)$

“... because if you've got a bleeding patient and we're at the operating table, I'm trying to deal with my bits, trying to fix the bleeding the ways I can, and so I rely on my anaesthetist to look at the ROTEM, interpret the ROTEM, and start to communicate what he thinks is the best way forward. It's absolutely collaborative. It can't be done any other way" (S)

"it's a multi-disciplinary program. To me, that is absolutely fundamental for the program having success, that everyone believes that they are part of the program they have developed, and they have ownership" (A)

"Listening to the conversations between the surgeons and anaesthetists.... for me, was the point in time when I thought this has a chance of working, of being sustainable" $(P)$

"We have discussions about what best based on evidence or not best guess. That's the advantage we have now, we can talk about the deficits are, how we are going to correct that, so we are empowered to discuss more about the best fit for the patient" (A)

"it's not the machines or the tests that make them (projects or programs) successful and make them right. It's the human interactions and the protocols and the building of teams that leads to success. I think the way to overcome the barriers is to build strong teams" (A) 
Motivation: Reflective Motivation, Automatic Motivation

'Motivation' (COM-B) refers to all the cerebral processes that direct behaviour, for example identifying with a professional role and evaluating potential consequences and benefits. Six barriers and five facilitators were categorised as influencing participants' motivation to manage bleeding or implement change to improve practice. These included three each related to "Belief about capabilities", and "Belief about consequences", and two each related to "Social professional roles and identity" and "Emotion".

\section{Belief about Capabilities (reflective motivation)}

Participants perceived a lack of confidence or familiarity with change or project management limited their ability to implement improvements.

"The project management skills that you need to set up a program are quite significant, and I think many of us, not only don't have the time to do it, but don't have the skills unless you have them in change management is a huge big deal. It's not just evidence, you know, it's leadership, it's bringing people on board, it's building a team behind you and getting that first four and the second four and moving forward. I think there is a lack of skill in change management. Intrinsically they're not there" (A)

"You virtually need a champion really; I don't know whether there would be resources within \#\#\#\#\#\#\# to support this. But it's a lot of work and it's a lot of effort and you've got to drive it and you've got to be persistent and dogged, and those are personality traits that don't universally exist" (A)

"... in other words, if you feel as though the action you are going to take is not really going to change practice or you don't feel like know how, or if you're not empowered to make change then you're less likely to even attempt to do so" $(P)$

Nevertheless, participants were confident that patients received the best care they could provide considering varied contexts, settings, as well as the resources they had available.

"So, between my anaesthetist and my perfusionist and myself, whether it's here or \#\#\#\#, you sort of cover most of your bases with the resources you have. Most of the people that I'm involved with either here, or next door are pretty knowledgeable about blood and roughly where the boundaries are for treatment, no treatment, so I think most of the bases are covered" (S)

"It's not a matter of going off and having a coffee and talking about it. It's finding the best solution in the immediate term and clearly sometimes that is a compromise" (A)

"We're not able to control or measure, we don't have a TEG or ROTEM, which means intellectually we've got an understanding of what the problem might be, but we have to make a decision based on the clinical 
environment in those situations. There isn't enough factual evidence to support every decision that you make" (A)

However, there was consensus by anaesthetists of a balance required to keep the surgeons "happy" and supported during difficult situations when patients were bleeding and potentially unstable. It was also considered that, as surgeons were responsible for overall patient outcomes, they were accountable in a final decision-making role. Consequently, anaesthetists felt less empowered to deliver interventions.

"At the end of the day, the surgeon's name is on the head of the bed and an ongoing problem that we have, is when I haven't done a ROTEM yet post-bypass that the surgeon just says, this patient is going to need platelets because they are on antiplatelets" (A)

"There have been times that I have just stopped transfusing. "Stop telling me it's a coagulopathy". It becomes a difficult position. You have got a bleeding patient and now you have got conflict and sometimes it's about for the safety of the patient, you just have to try and move past that conflict" (A)

"here it's definitely a team approach. I feel part of that team and I feel that I can use my knowledge to help come to a team decision but ultimately if a surgeon feels that there is a specific intervention that he or she would like me to do, then they are ultimately the ones managing the bleeding" (A)

".... there's an inherent need for the anaesthetist doing the list to keep the surgeon happy. The surgeon doesn't like the concept of blood conservation, then the anaesthetist would find it a struggle to put those things in place" (A)

"I try and monitor it all and bring it all together. At the end of the day, the surgeon's name is on the head of the bed" (A)

"I'm not going to say to a surgeon, don't use that product on a particular patient because they are at the point of treatment and I'm not" (A)

"there are some anaesthetists that work with some surgeons, mostly they follow their pattern of practice. So, it can be difficult sometimes applying all the guidelines" (A)

\section{Social professional roles and identity (reflective motivation)}

Several conflicting constructs around "social professional roles and identity" were evident. These centred around drivers for change, implementation of practice improvement, and the influence of the public or private setting. Anaesthesia were primarily perceived as the drivers to change practice however, there was consensus that success was dependent on surgeon 'buy-in'.

"The anaesthetists are really the ones who are the overarching drivers of blood management, the surgeons seemed to have embraced that as well... well they have to, or it won't work" $(P)$ 
"At an institution level, program level, I think anaesthesia probably have more involvement than surgery does" $(S)$

"It's more than just managing the bleeding, you have to manage the surgeon, perfusion, the situation, the environment as well as everything else going on with the patient. You learn this with time, some never learn" $(A)$

"If you've got the surgeon on board, everybody is on board in the whole process" (A)

"Well, intraoperatively it's the anaesthetic team that has a primary role because we're at the point of care. The surgeon is busy doing their highly skilled job and they are trying to stop as much bleeding as possible and minimise the amount but we're the ones who have got the full picture at that stage. We've got the clinical picture. We know what lab results are. We know what the status is of blood bank. We know the status is of cell saver and perfusion" (A)

"I think it's a collaborative approach, but I think it's mainly between surgeons and anaesthetics is the two biggest ones, however, I do think anaesthetics is the one who needs to take the role. In these situations, especially stressful situations with large amounts of bleeding, the surgeon is busy. They are operating. It's good to have their input but I think at that moment in time the anaesthetist is best suited to lead that charge" (A)

"I think surgeons........ it's hard for them because they are distracted by managing the bleeding surgically, they can't necessarily take an overall view at the time but in terms of strategies and hospital wide policies, there is no reason why a surgeon couldn't, here it's just been anaesthesia, we've done it" $(A)$

"I do actually because I think.... there are several reasons. One is that it's perioperative blood management. Anaesthetists are peri-op physicians, very well placed to do that. We often have a window to see our patients pre-operatively and it's a task that we can take, that honestly the surgeons would rather we did I think, because they have got plenty of other things to do" (A)

Participants provided insights into the social/professional differences and behaviours displayed by individuals operating in, or across the public and private sectors.

"Where this falls down (especially in the private sector) is the working relationship that the anaesthetist has to have with a surgeon, your private work is contingent on that" (A)

"It's different, in the private sector, I work with the same team all the time so it's a bit easier because my anaesthetist has a similar approach. In the public, it's not so easy from a staffing point of view because you're working with different people but in public, we have a better structure for what should happen to patients" (S)

"In the private sector, the surgeon definitely takes the lead because there is less of a system around you to manage blood and it's less easy, for instance, simple things like getting blood. You can wait hours for 
blood or platelets at some hospitals" (S)

"I work in a small institution that is private practice and I am responsible for my patients, so I have to take all the decisions" $(S)$

\section{Belief about Consequences (reflective motivation)}

Many participants reported differences in applying bleeding management strategies relating to private/public context with potential important consequences. Specifically, in the private sector, organisational culture meant that delaying surgery due to known bleeding risk (i.e. platelet dysfunction) was not desirable owing to financial implications, or the patient's desire not to delay surgery.

"There's a lot more pressure in the private sector, even though there is documented evidence of platelet disfunction, they'll still push for the patient to have surgery because there is a patient desire to get the surgery done, so they bleed, and they just give platelets" (A)

"So, the private centres are all competing with each other in order to get the surgery and the money, so they don't want to be the one that seemed to be delaying patients" (A)

"Their bottom line just comes down to dollars more than anything else. It has to be seen to be costing less money, or saving money in some aspect for it to be valid" $(P)$

Participants were not confident that implementing improvements bleeding management could be achieved with variable funding incentives such as the acquisition of blood products. The differences varied across states, and public and private hospitals.

"I believe that the expense that we can take in order to prevent bleeding would be more than mitigated by the outcomes of reduced bleeding, reduced transfusions, improved patients' haemoglobin postoperatively, the improved outcomes they would get, speedier recovery, 100\%" (A)

"It has a cost-benefit. It's harder to prove a cost-benefit to the hospital because we don't pay for blood. Blood is free. Everything else costs, blood management costs, cell salvage costs money. Trying to get cell salvage in and they just said, what's the cost benefit, how much will it cost? You try like a business case, but you can't use blood cost as a cost. You have to find other cost savings" (A)

"I would hate to be at the point which will happen soon, where we've made most of our cost savings from changing a transfusion practice and be wanting to implement other things that we know improve clinical care but come at a cost, it's going to be an uphill battle to try and argue for the cost, even though it's for better patient management" (A)

"Bleeding has to be managed well otherwise the consequences will be poorer outcomes. That is well established" (A) 
Participants consistently reported the belief that managing bleeding with evidence-based strategies provided benefits to the patient and the organisation.

"The other thing that is really fascinating about this, is that it's not just we're doing it here, its reproducible between institutions, because having fostered the same change in \#\#\#\# Hospital we actually have the equivalent outcome. So, this is a reproducible process" $(S)$

"There should be an expectation now that when you walk in for an elective operation that you walk out without having to be transfused. That will be the norm and it would be unusual to be transfused if you're doing everything in the bundle of care" (A)

"Because all of these blood related issues with regard to mortality and some morbidities are in the low percentages and can a long time to actually see a difference, a change or a positive benefit for patients and its only when they see it, that they can go "there is merit to this, it's not just anti transfusion, there is patient benefit, there is hospital benefit, there is financial benefit" $(P)$

"I knew that we could do better and if we did better, then we wouldn't have to take our patients back as much. So, that was actually what drove me to try and get a better understanding of what was going on" (A)

"The consequences of poor bleeding management are that we're most likely going to have more bleeding, poorer patient outcomes, and higher costs associated with it" (A)

\section{Emotion (automatic motivation)}

Participants unanimously reported that they were generally not troubled by emotion related to managing actual clinical bleeding.

"You know, a difficult case, there can be certain emotions around but that's generally, not usually a problem" (A)

"Not really, not more than any other aspect of clinical care" (A)

"No, emotions aren't helpful in difficult clinical situations" (A)

"Not really. I mean, it's part of our game" (A)

Participants did however, report frustration that evidence-based bleeding management strategies existed, and clinicians were not able to implement these strategies to improve patient care.

"If you can't get buy-in from any of the other teams, from management, it's just you, the lonely voice, it's hard" (A) 
"Administrative bureaucracy that prevents improvements from happening. That's one thing you have to overcome. I think that's definitely a big barrier, it's frustrating" (A)

\section{Discussion}

This study aimed to develop a comprehensive understanding of barriers and facilitators facing cardiac surgeons, anaesthetists and perfusionists implementing improved bleeding management practice in Australian cardiac surgery units, using theoretically grounded frameworks (TDF and COM-B model). We report important individual, social, and environmental barriers influencing clinician behaviour within this complex and under-reported reality;

\section{Capability;}

- lack of confidence with change management skills

- variability with non-technical skills

- lack of cross discipline cardiac surgery specific bleeding management education

\section{Opportunity}

- complicated institutional processes, including lack of organisational support

- lack of dedicated blood management clinicians (preferentially nursing)

- incongruent goals

\section{Motivation}

- disparities between public and private healthcare services

Key messages for enabling successful implementation were facilitating;

\section{Capability}

- standardisation,

- monitoring, auditing, and feedback of data,

- cross discipline training

\section{Opportunity}

- improved interpersonal and interdepartmental collaboration through shared goals

- efficient, supportive processes to allowing clinicians to navigate unfamiliar business and financial models of health care.

Our findings suggest as individuals, clinicians generally had the motivation to make change. It might seem unlikely that 'any' headway to improve care can be made by health care providers in the face of 
organisational complexity and bureaucracy, and yet it does. This body of work demonstrates there is strength in the bottom-up approach. In fact, health care teams are creative in response to complexity, often improving patient care, 'in spite' of the systems in which they work. The strategies are not always elegant, rather with an approach that negotiates competing demands, organisational hypocrisies and interprofessional tensions. It is important that healthcare organisations live up to their obligation and responsibility to partner with clinicians in supporting change and improving goal directed best practice.

Many variables of the healthcare work environment including infrastructure, staffing, equipment, and other resources were perceived to have a negative influence on clinicians 'physical opportunity' to implement change, with variables differing between public and private hospitals. Previous literature suggests these are not unique to bleeding management in cardiac surgery, with workload and time pressures often cited barriers to behaviour change (36).

Environmental factors can go beyond those tangible variables to include complicated and unfamiliar administrative processes, lack of proficiency and fluency with the development of quality initiatives, procedures, business cases that may be required for new equipment, therapies or additional clinical time $(37,38)$. A link was revealed between 'opportunity' and 'capability' with clinicians feeling restricted by their role delivering health care, and their capability to deliver behaviour change interventions echoed in data generated in this study. Furthermore, development of skills to deal with these organisational and environment issues are often not of interest to many medical professionals putting them at a disadvantage and poorly prepared to advance their change management goals (24). Awareness of the need and ability to improve these skills may also be related to the fact that implementation science is generally published in journals that anaesthetists, perfusionists and cardiac surgeons are less likely to read.

Results from this study revealed interplay with the constructs of physical 'opportunity' and 'capability'. Cross training and interprofessional education in health has been demonstrated to improve the delivery of care, a concept our participants believe would, in theory, be an important technique to improve decision-making across the surgical team (39-41). No participants were aware of an opportunity for this type of interprofessional education in Australia. Furthermore, creating a training program incorporating surgeons, anaesthetists and perfusion was judged unlikely to occur with presence the 'surgical' group who were perceived to consider themselves as 'ruggedly independent'. Indeed, there was a belief (not unanimous) that with 6 years of training, surgeons "knew how to manage bleeding". It is possible surgeons tend to downplay non-technical skills, regarding group training as a waste of their time. Methods to overcome this issue are likely to involve significant negotiating skills, as surgeons tend to perceive their leadership and communication skills requires no improvement $(42,43)$. These are significant issues as it is clearly reported that there are more incidents of preventable harm through poor management and leadership than clinical incompetence (44).

'Social opportunity' also has key role to play, as it is evident that while that cardiac surgery requires the collaboration of multiple expert clinicians with specific technical skills, non-technical skills are no less 
important. The social environment influencing effective relationships between clinical and non-clinical departments, including the alignment of goals and understanding of incentives and drivers, are known to be central to organisations delivering high quality care $(45,46)$. This study highlighted the need for support beyond the immediate clinical team with poor alignment of goals eroding clinicians' 'opportunity' to improve practice. This was particularly evident through a perceived absence of support from both public and private pathology, and haematology departments for viscoelastic haemostatic assays and platelet functions tests. These types of diagnostic assays are increasingly used to diagnose deficits in haemostatic capacity without which, treatment therapies are given 'blind' (47-52).

An identified lack of consistency of the team unit, particularly in public hospitals highlighted the use of standardised ways of working to improve clinician 'capability'. Decision support tools, checklists, protocols, and procedures are well documented to reduce variation in care and improve outcomes $(25,26$, $47-49,53)$. While the cardiac surgical team generally agreed with this concept; there was perceived need for autonomy in decision making. Clinical decision making is not always facts applied to a problem, and it was considered important to balance the use of standardisation and recommendations from guideline (considered to be aggregated needs of the cohort) with an intuitive, experiential approach, contextualising patient problems to determine best practice for the needs of the individual patient $(25,54)$.

A number of explanatory themes crosslinked with 'opportunity' and 'motivation' and vice versa. There was a level of frustration from clinicians regarding their capacity to full embrace evidenced-based bleeding management to improve outcomes for patients, and the organisations. Implementing change (specifically in the private sector) was complicated by the perception of pressure for surgery (despite risk of bleeding and potential requirement for blood transfusion) from; 1 . patients to get surgery done, and 2. the organisation not to cancel surgery. 'Motivation' for change was further complicated by the view from anaesthesia of a requirement to 'keep surgeons happy' to ensure ongoing private work. These issues are in conflict with the mandatory requirement to implement bleeding management strategies to achieve Australia's National Safety and Quality Health Service Standards (55). While this has been judged to drive organisational bleeding management change 'at a minimum', it was not considered to drive excellence $(25,26,49,54)$.

While there is currently little qualitative research on facilitators to implementing bleeding management in cardiac surgery in Australia, two studies have recently reported the benefits of monitoring, audit and feedback of data on outcomes and performance to reduce variability in practice $(25,26)$. This is also demonstrated in studies across other cohorts which also suggest capitalising on the driven and competitive behaviour of surgeons through regular analysis and feedback of data on outcomes and performance can be utilised as enabler to improve practice (56-60).

\section{Limitations}

The results from this interview study should be interpreted in the context of the combination of strengths and limitations innate with all research. While a broad cross-section of participants from varied hospitals 
and States/Territories were interviewed, findings may not have captured all relevant themes. Participants' observations and interpretations are subjective, and they may be biased in their viewpoints. However, the different interpretations and perceptions are undoubtedly part of the phenomena of this unique dynamic and must be highlighted. Further, the involvement of three researchers in the analysis improves the trustworthiness and credibility of the analysis; and the use of theoretically grounded frameworks facilitates comparisons between contexts, enabling theoretical generalisability. Strengths of this study include the variety of beliefs included member-checking to inform validity, and the achievement of data saturation across themes early in the analysis.

\section{Conclusion}

This study describes how factors associated with capability, opportunity and motivation were perceived by cardiac surgeons, anaesthetists and perfusionists and their ability to implement improvements in bleeding management practice. The findings suggest that while the knowledge and skills of this team are vast, the inclusion of the wider team has a significant impact. Although an obvious overall goal of those directly and indirectly involved would be positive patient outcomes, when specific aims are identified, not everyone's targets are aligned. Individual clinician barriers were identified as a lack of confidence with change management skills, variability with non-technical skills, lack of cross discipline cardiac surgery specific bleeding management education, complicated institutional processes, lack of dedicated blood management clinicians, incongruent goals, and disparities between public and private healthcare services. Key messages for enabling successful implementation were facilitating practice improvements were standardisation, monitoring, auditing, and feedback of data and efficient, supportive processes to allowing clinicians to navigate unfamiliar business and financial models of health care. What is clear from this study is that no one strategy can improve practice; success is dependent on 'mixing and matching' improvements of technical and non-technical skills and procedural and organisational measures, in conjunction with commitment to overarching shared goals. We now know, what needs to be done to support clinicians to close the knowledge practice gap; the next step is to formulate opportunities to make it happen.

\section{Declarations}

Ethics approval and consent to participate

The study approved by the Human Research Ethics Committees from The Prince Charles Hospital, (IRB No. HREC/17/QPCH/340), Griffith University (IRB No. 2017/590) and University of Sunshine Coast (IRB No. S171105); and carried out in accordance with the principles of the Declaration of Helsinki. Written informed consent was obtained from all participants.

Consent for Publication

Not applicable 
Availability of Data and Materials

The datasets used and/or analysed during the current study are available from the corresponding author on reasonable request.

Competing interests

Competitive grant funding to complete this study was received from the Australian National Blood Authority R\&D program. Griffith University has received unrestricted investigator-initiated research or educational grants on CMR's behalf from: BD-Bard and Cardinal Health). Griffith University has received consultancy payments on CMR's behalf from: 3M, BBraun, BD-Bard. SK's current and previous employer have received monies on her behalf from $3 \mathrm{M}$ and BD for research and educational consultancies, investigator-initiated grants, and unrestricted grants in aid. The authors report no other conflicts of interest in this work.

Funding

This study received funding from the Australian National Blood Authority Research \& Development program. The first author has also received a Griffith University Post-graduate research scholarship.

Author Contributions

All authors had input into the design of the study. BLP prepared the manuscript with contributions from SK, CMR and YLF. BLP, SK and YLF carried out the analysis and all reviewed the results. All authors read and approved the final manuscript.

Acknowledgements

The authors would like to acknowledge the contribution of the clinicians who participated in this research project.

\section{References}

1. Biancari F, Kinnunen EM, Kiviniemi T, Tauriainen T, Anttila V, Airaksinen JKE, et al. Meta-analysis of the Sources of Bleeding after Adult Cardiac Surgery. Journal of cardiothoracic and vascular anesthesia. 2018;32(4):1618-24.

2. Colson PH, Gaudard P, Fellahi JL, Bertet H, Faucanie M, Amour J, et al. Active Bleeding after Cardiac Surgery: A Prospective Observational Multicenter Study. PloS one. 2016;11(9):1-14.

3. Ruseckaite R, McQuilten ZK, Oldroyd JC, Richter TH, Cameron PA, Isbister JP, et al. Descriptive characteristics and in-hospital mortality of critically bleeding patients requiring massive transfusion: results from the Australian and New Zealand Massive Transfusion Registry. Vox sanguinis. 2017;112(3):240-8. 
4. Christensen MC, Krapf S, Kempel A, von Heymann C. Costs of excessive postoperative hemorrhage in cardiac surgery. The Journal of thoracic and cardiovascular surgery. 2009;138(3):687-93.

5. Al-Attar N, Johnston S, Jamous N, Mistry S, Ghosh E, Gangoli G, et al. Impact of bleeding complications on length of stay and critical care utilization in cardiac surgery patients in England. $J$ Cardiothorac Surg. 2019;14(1):1-10.

6. Ali JM, Wallwork K, Moorjani N. Do patients who require re-exploration for bleeding have inferior outcomes following cardiac surgery? Interactive cardiovascular and thoracic surgery. 2019;28(4):613-8.

7. Dixon B, Santamaria JD, Reid D, Collins M, Rechnitzer T, Newcomb AE, et al. The association of blood transfusion with mortality after cardiac surgery: cause or confounding? (CME). Transfusion. 2013;53(1):19-27.

8. Fröjd V, Jeppsson., Anders. Reexploration for Bleeding and Its Association With Mortality After Cardiac Surgery. Annals of Thoracic Surgery. 2016;102(1):109-17.

9. Ranucci M, Baryshnikova E, Castelvecchio S, Pelissero G, Surgical, Clinical Outcome Research G. Major bleeding, transfusions, and anemia: the deadly triad of cardiac surgery. The Annals of thoracic surgery. 2013;96(2):478-85.

10. Bhaskar B, Dulhunty J, Mullany DV, Fraser JF. Impact of blood product transfusion on short and longterm survival after cardiac surgery: more evidence. The Annals of thoracic surgery. 2012;94(2):460-7.

11. Cutrell JB, Barros N, McBroom M, Luby J, Minhajuddin A, Ring WS, et al. Risk factors for deep sternal wound infection after cardiac surgery: Influence of red blood cell transfusions and chronic infection. Am J Infect Control. 2016;44(11):1302-9.

12. Vranken NP, Weerwind PW, Barenbrug PJ, Teerenstra S, Ganushchak YM, Maessen JG. The role of patient's profile and allogeneic blood transfusion in development of post-cardiac surgery infections: a retrospective study. Interactive cardiovascular and thoracic surgery. 2014;19(2):232-8.

13. Horvath KA, Acker, M. A., Chang, H., Bagiella E, Smith PK, Iribarne A, Kron IL, Lackner P, et al. Blood transfusion and infection after cardiac surgery. The Annals of thoracic surgery. 2013;95(6):2194-201.

14. Task Force on Patient Blood Management for Adult Cardiac Surgery of the European Association for Cardio-Thoracic S, the European Association of Cardiothoracic A, Boer C, Meesters MI, Milojevic M, Benedetto U, et al. 2017 EACTS/EACTA Guidelines on patient blood management for adult cardiac surgery. Journal of cardiothoracic and vascular anesthesia. 2018;32(1):88-120.

15. Shore-Lesserson L, Baker RA, Ferraris VA, Greilich PE, Fitzgerald D, Roman P, et al. The Society of Thoracic Surgeons, The Society of Cardiovascular Anesthesiologists, and The American Society of ExtraCorporeal Technology: Clinical Practice Guidelines-Anticoagulation During Cardiopulmonary Bypass. Anesthesia and analgesia. 2018;126(2):413-24.

16. Kozek-Langenecker SA, Ahmed AB, Afshari A, Albaladejo P, Aldecoa C, Barauskas G, et al. Management of severe perioperative bleeding: guidelines from the European Society of Anaesthesiology: First update 2016. European journal of anaesthesiology. 2017;34(6):332-95. 
17. Society of Thoracic Surgeons Blood Conservation Guideline Task F, Ferraris VA, Brown JR, Despotis GJ, Hammon JW, Reece TB, et al. 2011 update to the Society of Thoracic Surgeons and the Society of Cardiovascular Anesthesiologists blood conservation clinical practice guidelines. The Annals of thoracic surgery. 2011;91(3):944-82.

18. Authority ANB. Patient blood management guidelines: Module 2 Perioperative: Australian National Blood Authority Canberra: Commonwealth of Australia; 2012 [Available from: http://www.blood.gov.au/pbm-module-2.

19. World Health Organisation (WHO). Availability, safety and quality of blood products: World Health Organisation World Health Organisation (WHO); 2010 [Available from: http://apps.who.int/gb/ebwha/pdf_files/WHA63/A63_R12-en.pdf.

20. Dixon B, Reid D, Collins M, Newcomb AE, Rosalion A, Yap CH, et al. The operating surgeon is an independent predictor of chest tube drainage following cardiac surgery. Journal of cardiothoracic and vascular anesthesia. 2014;28(2):242-6.

21. McQuilten ZK, Andrianopoulos N, Wood EM, Cole-Sinclair MF, McNeil JJ, Cameron PA, et al. Transfusion practice varies widely in cardiac surgery: Results from a national registry. The Journal of thoracic and cardiovascular surgery. 2014;147(5):1684-90 e1.

22. Karkouti K, Wijeysundera DN, Beattie WS, Callum JL, Cheng D, Dupuis JY, et al. Variability and predictability of large-volume red blood cell transfusion in cardiac surgery: a multicenter study. Transfusion. 2007;47(11):2081-8.

23. Jin R, Zelinka ES, McDonald J, Byrnes T, Grunkemeier GL, Brevig J, et al. Effect of hospital culture on blood transfusion in cardiac procedures. The Annals of thoracic surgery. 2013;95(4):1269-74.

24. Morrow G, Burford B, Redfern N, Briel R, Illing J. Does specialty training prepare doctors for senior roles? A questionnaire study of new UK consultants. Postgrad Med J. 2012;88(1044):558-65.

25. Pearse BL, Keogh S, Rickard CM, Faulke DJ, Smith I, Wall D, et al. Bleeding Management Practices of Australian Cardiac Surgeons, Anesthesiologists and Perfusionists: A Cross-Sectional National Survey Incorporating the Theoretical Domains Framework (TDF) and COM-B Model. J Multidiscip Healthc. 2020;13:27-41.

26. Pearse BL, Rickard CM, Keogh S, Lin Fung Y. A retrospective explanatory case study of the implementation of a bleeding management quality initiative, in an Australian cardiac surgery unit. Australian critical care : official journal of the Confederation of Australian Critical Care Nurses. 2019;32(2):92-9.

27. Delaforce A, Moore D, Duff J, Munday J, Hardy J. Assessing transfusion practice in elective surgical patients: a baseline audit. ISBT Science Series. 2019.

28. Michie S, van Stralen, M. M., West, R. The behaviour change wheel: A new method forcharacterising and designing behaviour changeinterventions. Implementation science : IS. 2011;6(42):1-11.

29. Cane J, O'Connor, D., Michie, S. Validation of the theoretical domains framework for use in behaviour change and implementation research. Implementation Science. 2012;7(37):1-17. 
30. Sargent L, McCullough A, Del Mar C, Lowe J. Using theory to explore facilitators and barriers to delayed prescribing in Australia: a qualitative study using the Theoretical Domains Framework and the Behaviour Change Wheel. BMC Fam Pract. 2017;18(1):1-13.

31. Flannery C, McHugh S, Anaba AE, Clifford E, O'Riordan M, Kenny LC, et al. Enablers and barriers to physical activity in overweight and obese pregnant women: an analysis informed by the theoretical domains framework and COM-B model. BMC Pregnancy Childbirth. 2018;18(1):178.

32. Cassidy C, Bishop A, Steenbeek A, Langille D, Martin-Misener R, Curran J. Barriers and enablers to sexual health service use among university students: a qualitative descriptive study using the Theoretical Domains Framework and COM-B model. BMC Health Serv Res. 2018;18(1):581.

33. Streeton R, Cooke, M., Campbell, J. Researching the researchers: using a snowball technique. Nurse Researcher. 2004;12(1).

34. Patey AM, Islam, R., Francis, J.J., Bryson, G.L., Grimshaw, J.M., . Anesthesiologists and surgeons perceptions about routine pre-operative testing in low-risk patients: application of the Theoretical Domains Framework (TDF) to identify factors that influence physicians decisions to order preoperative tests. Implementation Science. 2012;7(52).

35. Michie S, Atkins, L., \& West, R. . The behaviour change wheel: A guide to designing interventions. London, UK: Silverback; 2014.

36. Elwell L, Povey R, Grogan S, Allen C, Prestwich A. Patients' and practitioners' views on health behaviour change: a qualitative study. Psychol Health. 2013;28(6):653-74.

37. Andre B, Sjovold E. What characterizes the work culture at a hospital unit that successfully implements change - a correlation study. BMC Health Serv Res. 2017;17(1):486.

38. Jacobs SR, Weiner BJ, Reeve BB, Hofmann DA, Christian M, Weinberger M. Determining the predictors of innovation implementation in healthcare: a quantitative analysis of implementation effectiveness. BMC Health Serv Res. 2015;15:6.

39. Bruno. F.S., Hinthong W, Masamine J, Yasuoka J. Interprofessional Education for Whom? Challenges and Lessons Learned from Its Implementation in Developed Countries and Their Application to Developing Countries: A Systematic Review. PloS one. 2014;9(5).

40. Gilbert JHV, Yan, J., Hoffman, S.J. A WHO Report: Framework for Action on Interprofessional Education and Collaborative Practice. Journal of Allied Health. 2010;39(3):196-7.

41. Reeves S, Perrier L, Goldman J, Freeth D, Zwarenstein M. Interprofessional education: effects on professional practice and healthcare outcomes (update). The Cochrane database of systematic reviews. 2013(3):CD002213.

42. Wauben LSGL, Doorn CMD-v, Wijngaarden JDHv, Goossens RHM, Huijsman R, Klein J, et al. Discrepant perceptions of communication, teamwork and situation awareness among surgical team members. International Journal for Quality in Health Care. 2011;23(2):159 - 66.

43. Shubeck SP, Kanters AE, Dimick JB. Surgeon leadership style and risk-adjusted patient outcomes. Surg Endosc. 2019;33(2):471-4.

44. Management and Leadership - A Guide for Clincal Professionals. Switzerland: Springer; 2015. 
45. Wiegmann DA, Eggman AA, Elbardissi AW, Parker SH, Sundt TM, 3rd. Improving cardiac surgical care: a work systems approach. Appl Ergon. 2010;41(5):701-12.

46. Flin R, Patey R, Glavin R, Maran N. Anaesthetists' non-technical skills. British journal of anaesthesia. 2010;105(1):38-44.

47. Kuiper G, van Egmond LT, Henskens YMC, Roekaerts PM, Maessen JG, Ten Cate H, et al. Shifts of Transfusion Demand in Cardiac Surgery After Implementation of Rotational ThromboelastometryGuided Transfusion Protocols: Analysis of the HEROES-CS (HEmostasis Registry of patiEntS in Cardiac Surgery) Observational, Prospective Open Cohort Database. Journal of cardiothoracic and vascular anesthesia. 2019;33(2):307-17.

48. Karkouti K, Callum J, Wijeysundera DN, Rao V, Crowther M, Grocott HP, et al. Point-of-Care Hemostatic Testing in Cardiac Surgery: A Stepped-Wedge Clustered Randomized Controlled Trial. Circulation. 2016;134(16):1152-62.

49. Pearse BL, Smith I, Faulke D, Wall D, Fraser JF, Ryan EG, et al. Protocol guided bleeding management improves cardiac surgery patient outcomes. Vox sanguinis. 2015;109(3):267-79.

50. Wikkelso A, Wetterslev J, Moller AM, Afshari A. Thromboelastography (TEG) or rotational thromboelastometry (ROTEM) to monitor haemostatic treatment in bleeding patients: a systematic review with meta-analysis and trial sequential analysis. Anaesthesia. 2017;72(4):519-31.

51. Hans GA, Besser MW. The place of viscoelastic testing in clinical practice. $\mathrm{Br} \mathrm{J}$ Haematol. 2016;173(1):37-48.

52. Petricevic M, Biocina B, Milicic D, Konosic S, Svetina L, Lekic A, et al. Bleeding risk assessment using whole blood impedance aggregometry and rotational thromboelastometry in patients following cardiac surgery. Journal of thrombosis and thrombolysis. 2013;36(4):514-26.

53. Ali JM, Gerrard C, Clayton J, Moorjani N. Reduced re-exploration and blood product transfusion after the introduction of the Papworth haemostasis checklist. European journal of cardio-thoracic surgery : official journal of the European Association for Cardio-thoracic Surgery. 2019;55(4):729-36.

54. Delaforce A, Duff J, Munday J, Hardy J. Overcoming barriers to evidence-based patient blood management: a restricted review. Implementation science : IS. 2020;15(1):6.

55. ACSQHC. National-Safety-and-Quality-Health-Service-Standards, 2nd ed. In: Standards HC, editor. Sydney: Australian Commission of Safety and Qualtiy in Health Care; 2017.

56. Gould NJ, Lorencatto F, Stanworth SJ, Michie S, Prior ME, Glidewell L, et al. Application of theory to enhance audit and feedback interventions to increase the uptake of evidence-based transfusion practice: an intervention development protocol. Implementation science : IS. 2014;9:92.

57. Mehra T, Seifert B, Bravo-Reiter S, Wanner G, Dutkowski P, Holubec T, et al. Implementation of a patient blood management monitoring and feedback program significantly reduces transfusions and costs. Transfusion. 2015;55(12):2807-15.

58. Fischer DP, Zacharowski KD, Muller MM, Geisen C, Seifried E, Muller H, et al. Patient blood management implementation strategies and their effect on physicians' risk perception, clinical 
knowledge and perioperative practice - the frankfurt experience. Transfus Med Hemother. 2015;42(2):91-7.

59. Leahy MF, Roberts H, Mukhtar SA, Farmer S, Tovey J, Jewlachow V, et al. A pragmatic approach to embedding patient blood management in a tertiary hospital. Transfusion. 2014;54(4):1133-45.

60. Ierano C, Thursky K, Peel T, Rajkhowa A, Marshall C, Ayton D. Influences on surgical antimicrobial prophylaxis decision making by surgical craft groups, anaesthetists, pharmacists and nurses in public and private hospitals. PloS one. 2019;14(11):e0225011.

\section{Figures}

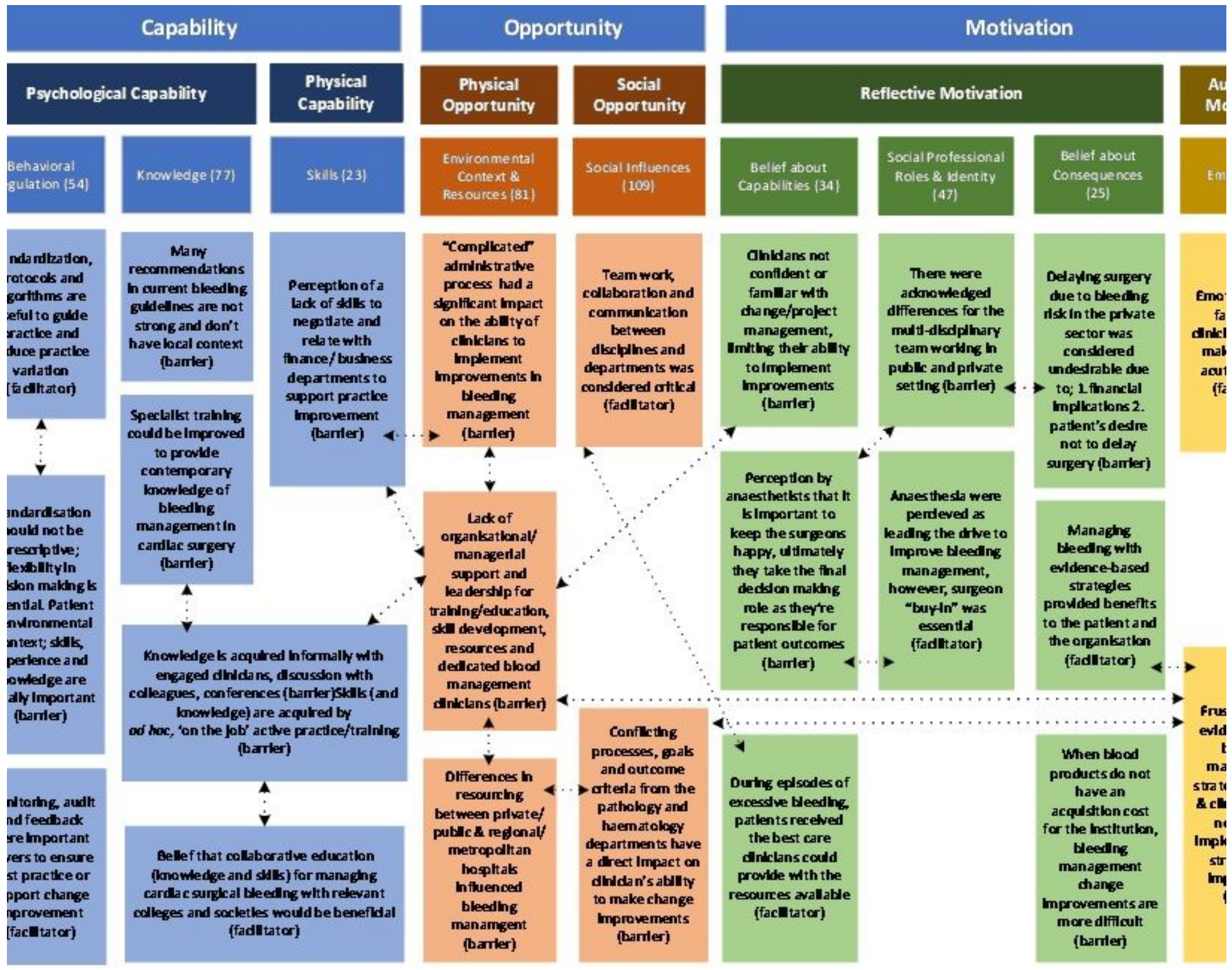

\section{Figure 1}

Explanatory themes describing the barriers and facilitators to practicing and implementing evidencebased bleeding management in Australian Cardiac Surgery Units. 